\title{
Blackbody-Radiation-Induced Decay and Excitation of Rydberg States in Sodium
}

\author{
V.D. Ovsiannikov and I.L. GluKhov* \\ Department of Physics, Voronezh State University, 394006, Voronezh, Russian Federation
}

\begin{abstract}
Rates of the blackbody radiation-induced excitation and decay were calculated for the $S$-, $P$-, $D$-series of the Rydberg states in sodium atoms with the Fues model potential method. Comparisons between three branches of the state depopulation mechanism (spontaneous decays, blakbody radiation-induced and excitations) were carried out. Simple analytical formulas were proposed for ratios of the blackbody radiation-induced decay and excitation rates to the spontaneous decay rate.
\end{abstract}

PACS numbers: $32.80 .-\mathrm{t}, 32.80 . \mathrm{Ee}, 32.70 . \mathrm{Cs}$

\section{Introduction}

Recent achievements in production of the Rydberg atoms stimulated worldwide investigations of highly excited states. On the one hand the interest to the Rydberg states originates from their dual nature as quantum objects with mesoscopic dimensions [1], on the other hand the Rydberg atoms have large dipole moments enabling strong interaction between neutral atoms which may be used in technological applications (for example, quantum processing of information [2]).

The basic characteristics of atomic states are their energies and lifetimes. It was pointed out first by Gallagher and Cooke [3] that blackbody radiation-induced (BBR-induced) transitions depopulate the Rydberg states with rates which are equal to or even exceed the spontaneous decay rates. Soon afterwards large-scale investigations of BBR influence on neutral atoms were performed [4-6] for highly excited states $|n l\rangle$ with principal quantum numbers $n$ up to 30. By the end of the millennium Rydberg states in neutral atoms attracted interests of investigators due to the following reasons: (i) general progress of experimental techniques (especially in the field of tunable lasers) [1]; (ii) exceptionally large electromagnetic susceptibilities and high sensitivity of Rydberg atoms to very weak external fields; (iii) wide spread of high-performance computers, which allowed to carry out considerable amounts of computation operations with an adequate accuracy within reasonable time intervals. In the last few years the interaction between BBR and Rydberg states with principal quantum numbers up to $n=100$ was in the focus, e.g. the BBR-induced transitions between bound levels [7], direct and stepwise ionization by $\operatorname{BBR}[8,9]$.

\footnotetext{
* corresponding author; e-mail: GlukhovOfficial@mail.ru
}

In overwhelming majority of papers on the BBR interaction with the Rydberg atoms, the BBR-induced transitions were considered all together (decay, excitation and ionization) as a depopulation factor for atomic states. In this paper we study the BBR-induced decays separately from the BBR-induced excitations for Rydberg states in sodium $S$-, $P$ - and $D$-series $(l=0,1,2)$. The calculated rates of induced by BBR decays and excitations, $P_{n l}^{\mathrm{d}}$ and $P_{n l}^{\mathrm{e}}$, are compared with the rates of spontaneous decays $P_{n l}^{\mathrm{sp}}$ by calculating the ratios

$$
R_{n l}^{\mathrm{d}(\mathrm{e})}=P_{n l}^{\mathrm{d}(\mathrm{e})} / P_{n l}^{\mathrm{sp}},
$$

which give an evident numerical information on the relative contribution of the BBR-induced transitions to the natural level width. The atomic units are used throughout the paper, if not otherwise specified explicitly.

2. Calculation of the decay and excitation rates

The radiation probabilities in the right-hand side of Eq. (1) are determined by the matrix elements of the dipole transitions from the Rydberg level $|n l\rangle$ to the lower (decay) and upper (excitation) bound states $\left|n^{\prime} l \pm 1\right\rangle$. In this paper, the wave functions of states are determined analytically on the basis of the Fues' model potential with some modification of parameters for $S$-states [9-11]. The model describes a valence electron in a field of atomic nucleus and electrons of the closed shells (so called core electrons). The Hamiltonian with the model potential for a valence electron has the form

$$
\hat{H}=-\frac{\nabla^{2}}{2}-\frac{Z}{r}+\sum_{l} \frac{B_{l}}{r^{2}} \hat{P}_{l},
$$

where $Z$ is a net charge of the nucleus and core electrons ( $Z=1$ for a neutral atom), $r$ is the distance between the valence electron and the atomic center, $\hat{P}_{l}$ is a projection operator onto the space of states with the given orbital quantum number $l$. The $l$-dependent numerical parameters $B_{l}$ determine the influence of the core electrons on the total potential and hence, on the wave functions of the valence electron. After separation of angular vari- 
ables, the Schrödinger equation for the radial wave functions $f_{n l}$ of a neutral atom $(Z=1)$ may be written as

$$
\left[\frac{\partial^{2}}{\partial r^{2}}-\frac{\lambda(\lambda+1)}{r^{2}}+\frac{2}{r}+2 E_{n l}\right]\left(r f_{n l}(r)\right)=0,
$$

where $\lambda$ is an effective orbital quantum number, which is determined from the centrifugal and core potential, as follows: $\lambda(\lambda+1)=l(l+1)+2 B_{l}$. The eigenvalue of Eq. (3) is the energy of a bound $|n l\rangle$-state

$$
E_{n l}=-\frac{1}{2 \nu_{n l}^{2}},
$$

where $\nu_{n l}=n_{\mathrm{r}}+\lambda+1, n_{\mathrm{r}}$ determines the number of the radial wave-function nodes (for the lowest state of a valence electron in $P$-, and $D$-series $n_{\mathrm{r}}=0$; for the lowest level in $S$-series $n_{\mathrm{r}}=1$ [11]). The corresponding eigenfunction is

$$
\begin{aligned}
& f_{n l}(r)=\frac{2}{\nu_{n l}^{2}} \sqrt{\frac{(2 \lambda+2)_{n_{\mathrm{r}}}}{n_{\mathrm{r}} ! \Gamma(2 \lambda+2)}} \exp \left(r / \nu_{n l}\right)\left(\frac{2 r}{\nu_{n l}}\right)^{\lambda} \\
& { }_{1} F_{1}\left(-n_{\mathrm{r}} ; 2 \lambda+2 ; \frac{2 r}{\nu_{n l}}\right),
\end{aligned}
$$

where $(a)_{n}=a(a+1) \cdot \ldots \cdot(a+n-1)$ is the Pochhammer symbol, ${ }_{1} F_{1}(a ; c ; x)$ is the confluent hypergeometric function. The parameter $\lambda$ is determined by Eq. (4) from the energy of the state $E_{n l}$ which may be taken from a database of atomic energy levels (see, for example [12]).

Wave functions (5) are suitable for calculations of the radial dipole matrix elements

$$
M_{n l \rightarrow n^{\prime} l^{\prime}}=\int_{0}^{\infty} f_{n^{\prime} l^{\prime}}(r) r f_{n l}(r) r^{2} \mathrm{~d} r,
$$

which determine the spontaneous decay rate, as follows:

$$
\begin{aligned}
& P_{n l}^{\mathrm{sp}}=\frac{4 \alpha^{3}}{3(2 l+1)} \sum_{n^{\prime}}^{E_{n^{\prime} l \pm 1}<E_{n l}} \\
& \omega_{n n^{\prime}}^{3}\left(l M_{n l \rightarrow n^{\prime} l-1}^{2}+(l+1) M_{n l \rightarrow n^{\prime} l+1}^{2}\right),
\end{aligned}
$$

where $\alpha=1 / 137.036$ is the fine-structure constant, $\omega_{n n^{\prime}}=E_{n l}-E_{n^{\prime} l \pm 1}$ is the frequency of transition from the Rydberg state $|n l\rangle$ to a state $\left|n^{\prime} l \pm 1\right\rangle$ with lower energy $E_{n^{\prime} l \pm 1}<E_{n l}$. For the BBR-induced decay rate a similar expression holds, with each term times the number density of BBR photons at the ambient temperature $T$, determined by the Planck distribution

$$
\begin{aligned}
& P_{n l}^{\mathrm{d}}=\frac{4 \alpha^{3}}{3(2 l+1)} \sum_{n^{\prime}}^{E_{n^{\prime} l \pm 1}<E_{n l}} \\
& \frac{\omega_{n n^{\prime}}^{3}\left(l M_{n l \rightarrow n^{\prime} l-1}^{2}+(l+1) M_{n l \rightarrow n^{\prime} l+1}^{2}\right)}{\exp \left[\omega_{n n^{\prime}} /(k T)\right]-1},
\end{aligned}
$$

where $k=3.1668 \times 10^{-6}$ a.u. $/ \mathrm{K}$ is the Boltzmann constant. Equation (8) with summation over infinite number of bound states above the state $|n l\rangle$ (i.e. over states $\left|n^{\prime} l \pm 1\right\rangle$ with $\left.E_{n^{\prime} l \pm 1}>E_{n l}\right)$ holds also for the BBR-induced excitation rate $P_{n l}^{\mathrm{e}}$.

The wave functions (5) allow analytical integration in Eq. (6):

$$
\begin{aligned}
& M_{n l \rightarrow n^{\prime} l^{\prime}}=\frac{1}{4} \frac{\Gamma\left(\lambda+\lambda^{\prime}+4\right)}{\sqrt{\Gamma(2 \lambda+2) \Gamma\left(2 \lambda^{\prime}+2\right)}} \\
& \quad \times \sqrt{\frac{(2 \lambda+2)_{n_{\mathrm{r}}}}{n_{\mathrm{r}} !} \frac{\left(2 \lambda^{\prime}+2\right)_{n_{\mathrm{r}}^{\prime}}}{n_{\mathrm{r}}^{\prime} !} x_{1}^{\lambda+2} x_{2}^{\lambda^{\prime}+2}}
\end{aligned}
$$$$
\times F_{2}\left(\lambda+\lambda^{\prime}+4,-n_{\mathrm{r}},-n_{\mathrm{r}}^{\prime} ; 2 \lambda+2,2 \lambda^{\prime}+2 ; x_{1}, x_{2}\right),(9)
$$

where $F_{2}\left(a, b_{1}, b_{2} ; c_{1}, c_{2} ; x_{1}, x_{2}\right)$ is the generalized hypergeometric function of two variables $x_{1}=2 \nu^{\prime} /\left(\nu+\nu^{\prime}\right)$ and $x_{2}=2 \nu /\left(\nu+\nu^{\prime}\right)$, which may be presented as a twofold sum of $\left(n_{\mathrm{r}}+1\right) \cdot\left(n_{\mathrm{r}}^{\prime}+1\right)$ terms with alternating signs. Due to a strong cancellation of digits, the calculation of the matrix element (9) requires a high-precision presentation of the terms in $F_{2}$, with corresponding extension of the digit number. Calculations demonstrate a good agreement of the numerical values for the radial matrix elements (9) with corresponding values given by formulae of the quasi-classical WKB approximation in the quantum defect method [13], which works but only in the case of very large values of both $\nu$ and $\nu^{\prime}$, so that $\left|\nu-\nu^{\prime}\right| \ll \nu \approx \nu^{\prime}$. Meanwhile expression (9) holds for arbitrary values of $\nu$ and $\nu^{\prime}$ and this feature is very important, since the principal contribution to the sum (7) (and (8) at rather high temperatures $T>(100 / \nu)^{3} \mathrm{~K}$ ) comes from states with $\left|\nu-\nu^{\prime}\right| \approx \nu$.

The values of the ratios (1) are strongly dependent both on the principal quantum number and on the ambient temperature. However, the general property holds for all the series of states: the rates of the decay and excitation are nearly equal to one another and both of them essentially exceed the ionization rate.

Matrix elements necessary for estimations of the rates (8) of the BBR-induced decays and excitations in $S$-, $P$ and $D$-series of sodium Rydberg states were determined and stored for calculations at different temperatures. The results at $T=300 \mathrm{~K}$ are in a good agreement with those of the papers $[4,5,7]$.

\section{Approximations for ratios of induced and spontaneous rates}

The calculated rates (8) of the BBR-induced decays and excitations for the Rydberg states were compared with corresponding spontaneous decay rates which may be scaled as $P_{n l}^{\mathrm{sp}} \sim \nu^{-3}[5,14]$. Asymptotic approximations for rather high $\nu_{n l}$ may be proposed for the rates (8), in analogy with that for the BBR-induced ionization rate [10]. Numerical calculations demonstrate that rather simple and accurate approximations hold also for the ratios (1).

The rates in numerator and denominator of the fraction (1) decrease when $\nu \rightarrow \infty$, but their ratio will increase, so the contribution of the closest states $\left|n^{\prime} l \pm 1\right\rangle$ in the sum (8) with $\nu \approx \nu^{\prime}$ is emphasized in $R_{n l}^{\mathrm{d}(\mathrm{e})}$. Therefore, the transition frequency in the exponent of the Planck distribution may be estimated as $\omega_{n n^{\prime}} \approx 1 / \nu_{n l}^{3}$ and the most reasonable approximation may be written as 


$$
R_{n l}^{\mathrm{d}(\mathrm{e})}=\frac{a_{0}^{\mathrm{d}(\mathrm{e})}+a_{1}^{\mathrm{d}(\mathrm{e})} x+a_{2}^{\mathrm{d}(\mathrm{e})} x^{2}}{\nu^{2}\left[\exp \left(0.31579 x^{3}\right)-1\right]},
$$

where $x=100 /\left(\nu_{n l} T^{1 / 3}\right)$ is a dimensionless parameter with the temperature $T$ measured in kelvin, the number in exponent comes from the Boltzmann constant in atomic units. From this presentation one can see that for large $\nu$ and $T$, when the $x$-parameter vanishes, the ratio (10) becomes directly proportional to the product of both parameters

$$
R_{n l}^{\mathrm{d}(\mathrm{e})} \approx 3.1666 \times 10^{-6} a_{0}^{\mathrm{d}(\mathrm{e})} \cdot \nu_{n l} T .
$$

Figure 1 illustrates the precision of approximation (10) for the numerical values of the ratio $R_{n l}^{\mathrm{e}}$ at $T=300 \mathrm{~K}$ in $n P$-series of $\mathrm{Na}$, in comparison with the data of straightforward calculations according to Eqs. (7)-(9). In particular, the linear dependence (11) can be seen in the figure already for $\nu>20$.

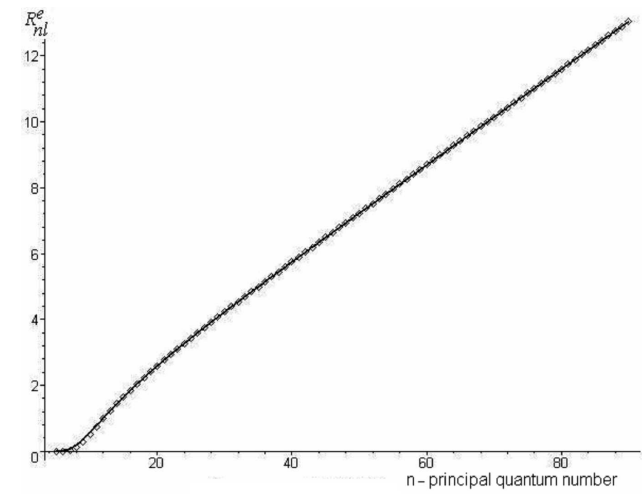

Fig. 1. Relative BBR-induced excitation rates $R_{n l}^{\mathrm{e}}$ for P-series of $\mathrm{Na}$ at $T=300 \mathrm{~K}$. Full line represents approximation (10); diamonds - results of direct calculations according to Eqs. (7)-(9).

Further analysis demonstrated that the coefficients $a_{i}^{\mathrm{d}(\mathrm{e})}$ of the quadratic polynomial in Eq. (10) slightly depend on temperature. To fit this dependence, the second-order polynomial in powers of inverse absolute temperature $T$ was proposed

$$
a_{i}^{\mathrm{d}(\mathrm{e})}(T)=\sum_{k=0}^{2} b_{i k}^{\mathrm{d}(\mathrm{e})}(100 / T)^{k}, \quad i=0,1,2,
$$

which provides an accurate parameterization for the BBR temperatures $T>100$.

\section{Results and discussions}

On the basis of the fitting procedure for the computed numerical data of the ratio (1) the $a_{i}^{\mathrm{d}(\mathrm{e})}$-coefficients of Eq. (10) were determined for $n S$-, $n P$ - and $n D$-series in sodium at different temperatures. The subsequent processing of the numerical data for $a_{i}^{\mathrm{d}(\mathrm{e})}$-coefficients resulted in the formation of matrices of the fitting coefficients $b_{i k}^{\mathrm{d}(\mathrm{e})}$ of Eq. (12), which are presented below.

\subsection{Modification of approximation (10) for $n$ S-states}

As $S$-states may decay or excite only into $P$-states, it is possible to make $T$-independent the coefficients $a_{i}^{\mathrm{d}(\mathrm{e})}$ of Eq. (10) by introducing an additional factor $\Delta_{\mu}^{\mathrm{d}(\mathrm{e})}$ to the argument of the Planck-distribution exponent

$$
R_{n l}^{\mathrm{d}(\mathrm{e})}=\frac{a_{0}^{\mathrm{d}(\mathrm{e})}+a_{1}^{\mathrm{d}(\mathrm{e})} x+a_{2}^{\mathrm{d}(\mathrm{e})} x^{2}}{v^{2}\left[\exp \left(0.31579 \Delta_{\mu}^{\mathrm{d}(\mathrm{e})} x^{3}\right)-1\right]},
$$

where $\Delta_{\mu}^{\mathrm{d}(\mathrm{e})}$ is determined by the quantum defects $\mu_{\mathrm{S}}$ and $\mu_{\mathrm{P}}$ of the $S$ - and $P$-series of the Rydberg states. Thus, $\Delta_{\mu}^{\mathrm{d}}=\mu_{P}-\mu_{S}+1$ for decays and $\Delta_{\mu}^{\mathrm{e}}=\mu_{S}-\mu_{P}$ for excitations provide a more exact account for the energy separation between the neighbour $n S$ and $n P$ states with the energies $E_{n l}=-1 /\left[2\left(n-\mu_{l}\right)^{2}\right]$. It was found that for $n S$-states the coefficients $a_{i}^{\mathrm{d}(\mathrm{e})}$ in (12) may be taken constant for $T>100 \mathrm{~K}$, as follows:

$$
\begin{aligned}
& a_{0}^{\mathrm{d}}=8.5833, \quad a_{1}^{\mathrm{d}}=-2.8473, \quad a_{2}^{\mathrm{d}}=0.2966, \\
& a_{0}^{\mathrm{e}}=7.1104, \quad a_{1}^{\mathrm{e}}=-1.4804, \quad a_{2}^{\mathrm{e}}=0.4297 .
\end{aligned}
$$

The values of coefficients $a_{0}^{\mathrm{d}}$ and $a_{0}^{\mathrm{e}}$ indicate, for example, that from rather high $n S$-levels and temperatures the decay processes (BBR-induced together with spontaneous) dominate over the BBR-induced excitation, so the lower $P$-states are populated rather than the upper states.

\subsection{The constants of Eqs. (10) and (12) for nP states}

The spontaneous decay rate of $P$-states is one order smaller than that of the $S$-states [5, 14], therefore the decay and excitation ratios (1) exceed those for the $n S$ series by more than one order, and the corresponding coefficients $b_{i k}^{\mathrm{d}(\mathrm{e})}$ have relatively large values (at $T=300 \mathrm{~K}$ $R_{n l}^{\mathrm{e}}>1$ already for $n=12$ and $R_{n l}^{\mathrm{d}}>1$ for $\left.n \geq 17\right)$ :

$$
\begin{aligned}
b_{i k}^{\mathrm{e}} & =\left(\begin{array}{ccc}
151.130 & 0.678 & -0.329 \\
32.933 & -46.668 & 27.353 \\
-46.653 & 94.481 & -59.850
\end{array}\right), \\
b_{i k}^{\mathrm{d}} & =\left(\begin{array}{ccc}
107.636 & 5.943 & -3.972 \\
-83.537 & 54.087 & -34.275 \\
39.861 & -26.842 & 17.897
\end{array}\right) .
\end{aligned}
$$

Here the coefficients $b_{00}^{\mathrm{d}}$ and $b_{00}^{\mathrm{e}}$ indicate a more rapid population of upper $S$ - and $D$-states from the thermal excitation Rydberg $P$-states.

\subsection{The constants of Eqs. (10) and (12) for nD states}

The spontaneous decay rates of $D$-states almost coincide with those of the $S$-states $[5,14]$. Therefore, the ratios (1) for $D$-states are nearly equivalent to those of $S$-states, as it follows from the matrices of coefficients $b_{i k}^{\mathrm{d}(\mathrm{e})}$ :

$$
b_{i k}^{\mathrm{d}}=\left(\begin{array}{ccc}
11.546 & 0.309 & 0.101 \\
-11.085 & 1.948 & -0.571 \\
4.311 & -1.851 & 0.577
\end{array}\right) \text {, }
$$




$$
b_{i k}^{\mathrm{e}}=\left(\begin{array}{ccc}
5.232 & -0.2667 & 0.4507 \\
0.1574 & -0.1713 & -1.277 \\
0.2334 & -3.5013 & 3.0608
\end{array}\right) \text {. }
$$

\section{Conclusions}

Comparison of the data given by Eqs. (10) and (12) with that of the straightforward calculations according Eqs. (7)-(9) demonstrates high accuracy of the asymptotic approximations within 1-2\% for the states with $n$ from 12 up to $n=1000$ in the temperature ranges from $T=100 \mathrm{~K}$ up to $10000 \mathrm{~K}$. The proposed approximation equations may be derived also for the Rydberg states in other atoms.

\section{Acknowledgments}

This work was supported by the Russian Foundation for the Basic Research (RFBR) grant No. 07-02-00279-a.

\section{References}

[1] F.B. Dunning, J.J. Mestayer, C.O. Reinhold, S. Yoshida, J. Burgdorfer, J. Phys. B 42, 022001 (2009).
[2] I.I. Ryabtsev, D.B. Tretyakov, I.I. Beterov, J. Phys. B 38, S421 (2005).

[3] T.F. Gallagher, W.E. Cooke, Phys. Rev. Lett. 42, 835 (1979).

[4] J.W. Farley, W.H. Wing, Phys. Rev. A 23, 2397 (1981).

[5] C.E. Theodosiou, Phys. Rev. A 30, 2881 (1984).

[6] C.E. Theodosiou, Phys. Rev. A 30, 2910 (1984).

[7] I.I. Beterov, I.I. Ryabtsev, D.B. Tretyakov, V.M. Entin, Phys. Rev. A 79, 052504 (2009).

[8] I.I. Beterov, D.B. Tretyakov, I.I. Ryabtsev, V.M. Entin, A. Ekers, N.N. Bezuglov, New J. of Phys. 11, 013052 (2009).

[9] N.L. Manakov, V.D. Ovsiannikov, L.P. Rapoport, Phys. Rep. 141, 319 (1986).

[10] I.L. Glukhov, V.D. Ovsiannikov, J. Phys. B 42, 075001 (2009).

[11] A.A. Kamenski, V.D. Ovsiannikov, J. Phys. B 39, 2247 (2006)

[12] http://physics.nist.gov/asd3 .

[13] V.A. Davydkin, B.A. Zon, Opt. Spektr. 51, 25 (1981).

[14] V.D. Ovsiannikov, I.L. Glukhov, Proc. Voronezh State Univ. Ser. Physics Math. 2, 99 (2006). 